Seguridad Operacional

y Logística Aeronáutica

Segurança Operacional

e Logística Aeronáutica

Operational Safety

and Aviation Logistics

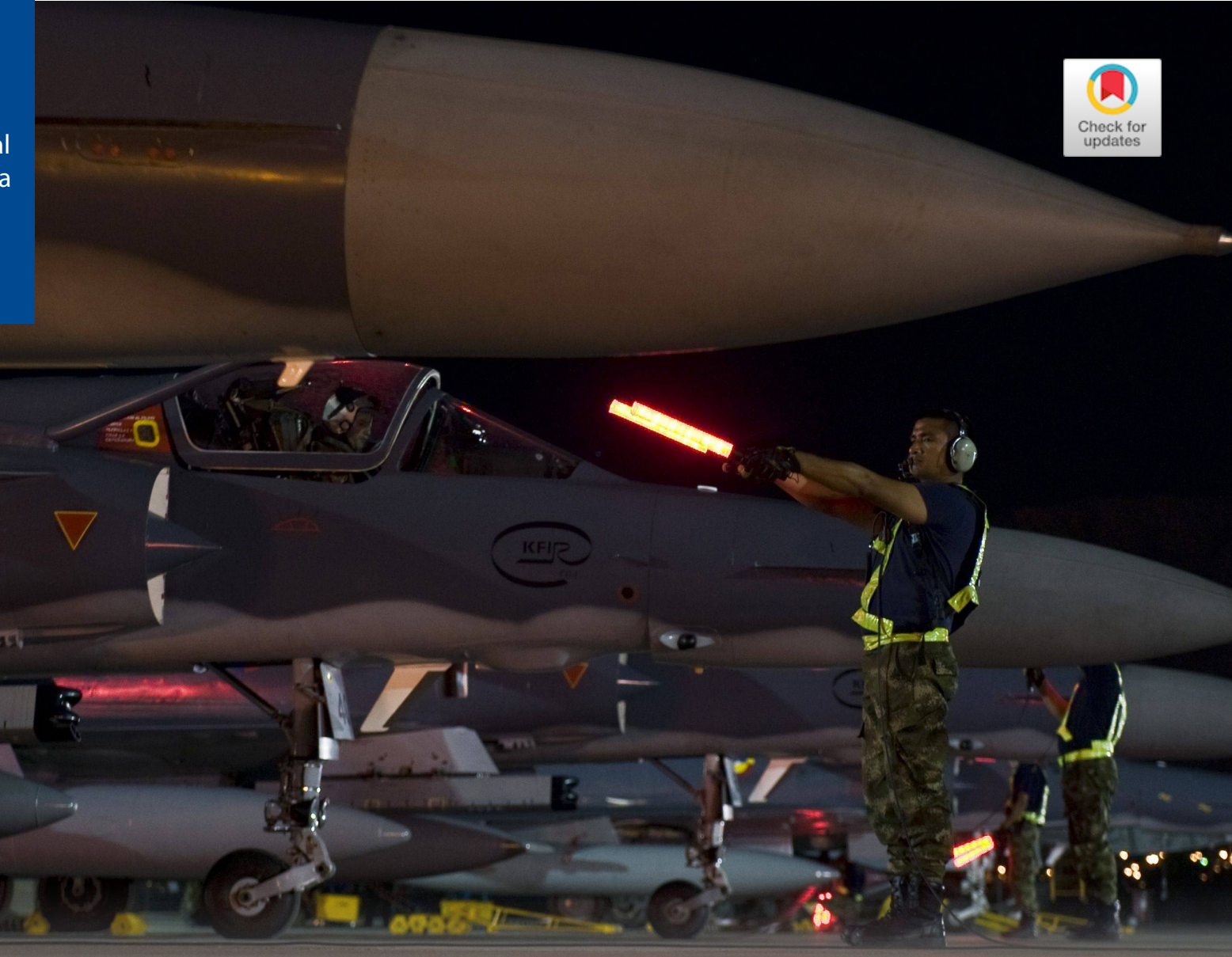

\title{
Caracterización del Clúster aeroespacial en el Valle del Cauca aplicando el méto- DO CUALITATIVO QUE PERMITA IDENTIFICAR LAS ESTRATEGIAS DE GESTIÓN COMPETIVAS*
}

CaracterizaÇão do Cluster aeronáutico de Valle del Cauca aplicaÇão do método Qualitativo que autorizaÇão O ESTRATÉGIAS GESTÃO COMPETITIVA *

Characterization of the Aerospace Cluster in the Valle del Cauca Applying the Qualitative Method that Permits IDENTIFYING the Competitive Management StRateGies ${ }^{* * *}$

Diego Fernando Morante Granoblesª y Juan Pablo Hernández Narváez ${ }^{b}$ Escuela Militar de Aviación Marco Fidel Suárez. Bogotá, Colombia

\section{CIENCIA Y PODER AÉREO}

ISSN 1909-7050 / E- ISSN 2389-2468 / Volumen 12/ Enero-diciembre de 2017/ Colombia/ Pp. 60-70 Recibido: 21/11/2016

Aprobado: 10/12/2016

Doi: https://doi.org/10.18667/cienciaypoderaereo.561 
Resumen: este trabajo se centra en la documentación del estado actual de las empresas, academia y entidades gubernamentales que integran el Clúster Aeroespacial del Valle del Cauca. A través del análisis de esta documentación, utilizando el método cualitativo -en cuanto a las entrevistas realizadas a los actores expertos del sector, seleccionados por su conocimiento y experien-

Para citar este artículo:

Hernández, J., \& Morante, D. (2016). Caracterización del clúster aeroespacial en el Valle del Cauca aplicando el método cualitativo que permita identificar las estrategias de gestión competivas. Ciencia y Poder Aéreo, 12, 60-70. Doi: https://doi.org/10.18667/cienciaypoderaereo.561

* Artículo de reflexión derivado del proyecto de investigación para tesis doctoral del profesor Diego Fernando Morante Granobles, titulado «Tecnología e Innovación en el diseño de un modelo de gestión para el desarrollo del clúster aeroespacial del Valle del Cauca (Colombia)».

** Artigo de reflexão derivado do projeto de pesquisa de doutorado do professor Diego Fernando Morante Granobles, intitulado "Tecnologia e inovação no projeto de um modelo de gestão para o desenvolvimento do cluster aeroespacial de Valle del Cauca (Colômbia)".

${ }^{* * *}$ Reflection article derived from research project for doctoral thesis Professor Diego Fernando Morante Granobles, entitled "Technology and Innovation in the design of a management model for the development of the aerospace cluster of Valle del Cauca (Colombia)."

a TE.Rva. PhD. Gestión Tecnológica e Innovación, magíster en Ingeniería Industrial, especialista en Gestión Tecnológica e Innovación, especialista en Docencia para la Educación Superior, administrador de Empresas, docente de la Escuela Militar de Aviación Marco Fidel Suárez. Cali, Colombia. Correo electrónico: dfmorante@emavirtual.edu.co.

b Estudiante de Administración Aeronaútica. Escuela Militar de Aviación Marco Fidel Suárez. Cali, Colombia. Correo electrónico: juanpa9960@gmail.com. cia-, y por medio de la utilización de la matriz DOFA, se realiza el diagnóstico del estado actual en el que se encuentra el sector. Lo anterior permite la identificación de las estrategias competitivas que servirán de aporte, como parte de la asistencia de investigación, a la tesis doctoral titulada «Tecnología e innovación en el diseño de un modelo de gestión para el desarrollo del clúster aeroespacial del Valle del Cauca (Col)», que busca consolidar una propuesta de gestión para el desarrollo del clúster aeronáutico en Valle del Cauca.

Palabras clave: aeroespacial, clúster, competitividad, cualitativo, gestión.

Resumo: O objetivo deste trabalho centrou-se na documentação do estado atual dos negócios, academia e entidades governamentais que compõem o cluster Aeroespacial do Valle del Cauca, através da análise e síntese usando o método qualitativo através de entrevistas com especialistas jogadores selecionados para seu conhecimento e experiência com o diagnóstico sector informação obtida e análise do estado atual em que é o setor por meio do uso da matriz SWOT é realizada, permitindo a identificação de estratégias competitivas que alimentarão como parte da assistência de pesquisa da tese de doutoramento intitulada "Tecnologia e Inovação na concepção de um modelo de gestão para o desenvolvimento do Aerospace Cluster de Valle del Cauca (Col)" para consolidar a proposta da administração para o desenvolvimento de clusters Aeronáutico no Valle del Cauca.

Palavras-chave: Aerospace, Cluster, Competitividade, Qualitativa, Gestão.

Abstract: This article presents the implementation of aeronautical technologies to meet the immediate need that the climate changes raising the urgency in the acquisition of a fleet of airplanes to extinguish forest fires. For this, the Colombian State must ensure the implementation of technologies that allow the application of a dynamic solution according to the detected contingencies, in a scenario of reasonable risk management, with effective support in the coverage of the national territory, to Colombian Firemen in critical and dangerous situations. The objective evidences, situations and data presented will allow us to understand the immediate need to be met by the State, in the permanent search for a more humane nation.

Key Words: Agroforestry disaster; Air Tractor AT-802; environmental risk; forest fire; Reforestation and restoration. 


\section{Introducción}

La presente investigación trata la situación actual del Clúster Aeroespacial en el Valle del Cauca, partiendo del estudio de los aciertos y desaciertos de los agentes involucrados, directa o indirectamente, en la parte operacional. Dada la situación de bajo nivel organizacional que el sector ha venido presentando últimamente, el estudio ofrece un panorama general, identificando las variables que se deben mantener y aquellas que hay que corregir. La información obtenida determinó las posibles medidas para reactivar el clúster aeroespacial en el Valle del Cauca.

La historia de la aviación en Colombia se remonta a los inicios del siglo XX, constituyéndose como pionera a nivel continental en la utilización de este medio de transporte que, con el transcurrir de los años, fue creciendo simultáneamente en el ámbito militar y en el civil. Sin embargo, la aviación en Colombia tenía como base fundamental la industria extranjera; a nivel nacional no se introdujo en este sector, lo que llevó a que el 100\% de las aeronaves tuvieran que ser importadas. Este es un hecho paradójico ya que Colombia es un país que siempre ha tenido una cantidad bastante significativa de operaciones aéreas, o casi igual de grande a otros países como Brasil, Argentina y Chile, que tienen una industria aeronáutica mucho más avanzada a la del país.

Sin embargo, la industria aeronáutica colombiana ha ido avanzando en el ámbito militar y en el de ultralivianos, debido a que en el primer caso tiene el apoyo del Estado -a través de la Fuerza Aérea Colombiana y las unidades de aviación de las otras fuerzas-, y en el segundo caso, un par de empresas han logrado tener cierto éxito en el sector aeronáutico comercial, dado que el marco legal que les rige es más flexible y las regulaciones del RAC (Reglamentos Aéreo Colombiano) no son tan estrictas.

Por otro lado, en las últimas décadas se ha empezado a desarrollar la industria del mantenimiento de aeronaves, pues prevalece la necesidad de las compañías aéreas y del Estado de tener un alto grado de alistamiento de sus aeronaves. El problema para estas empresas ha sido la carencia de un marco legal detallado y completo que les permita tener un panorama claro acerca de cuál es el paso para que estas autopartes sean autorizadas y certificadas por la Aéreocivil y cumplan con los requisitos exigidos por el RAC y la OACI (Organización de Aviación Civil Internacional).

El 23 de noviembre del 2011 se lanzó oficialmente el Clúster Aeroespacial en el Valle del Cauca con el apoyo de la Fuerza Aérea Colombiana, a través del CITAE (Centro de Investigación de Tecnologías Aeroespaciales). El clúster no ha tenido el éxito esperado después de cuatro años de creación, por la falta de voluntad y confianza entre la mayoría de empresarios involucrados, lo que ha hecho que se desperdicie la posibilidad de involucrarse en un mercado que crece cada vez más y que se puede desarrollar en gran medida en una región tan privilegiada geográficamente como lo es el Valle del Cauca. En el caso específico de la Fuerza Aérea Colombiana, se está perdiendo la posibilidad de tener un grupo de proveedores que le puedan brindar repuestos y servicios de mantenimiento para sus aeronaves, lo que llevaría a un ahorro de costos y tiempo en el alistamiento de sus aeronaves.

El propósito de este trabajo es realizar un estudio cualitativo (Hernández et àl., 2010) a través del análisis acerca del estado actual e del clúster aeroespacial en el Valle del Cauca, realizando entrevistas a personas involucradas Clúster, de los resultados en las entrevistas y el conversatorio, se elabora e la DOFA identificando las estrategias competitivas que servirán de aporte de la asistencia de investigación para la Tesis Doctoral titulada "Tecnología e Innovación en el diseño de un Modelo de Gestión para el Desarrollo del Clúster Aeroespacial del Valle del Cauca (Colombia)".

Es importante mencionar que este estudio tiene como base teórica las ventajas tanto comparativas como competitivas que poseen los diferentes clústeres aeronáuticos alrededor del mundo y el cómo los han desarrollado y sostenido; dentro de este se incluye la teoría de la competitividad de Michael Porter (2007) que es referente para el análisis de clúster a través de las fuerzas competitivas y del funcionamiento de los mercados en el mundo.

\section{Revisión Literaria}

El marco referencial presenta como base conceptual el término clúster y el tipo de estudio cualitativo.

En primer lugar el término clúster se define como «una concentración sectorial y/o geográfica de empresas que se desempeñan en las mismas actividades o en actividades estrechamente relacionadas tanto hacia atrás, proveedores de insumos y equipos, como hacia delante y hacia los lados» (Perego, 2003, p. 2), además, busca «crear un núcleo de servicios comunes para las empresas e integrarlos en una cadena de valor productiva y, que en conjunto, alcancen más altos niveles de productividad, calidad y rentabilidad» (Perego, 2003, p. 69).

Igualmente analizando el diamante competitivo (Porter, 2007, pag.69-95) este constituye una serie de atributos como son:

La condición de los factores se refiere al estado en el que se encuentra la agrupación industrial o la compañía como la 
infraestructura, el desarrollo tecnológico, la mano de obra calificada entre otras que influyen fuertemente en el nivel de competitividad que pueda desarrollar una compañía.

Con respecto a las condiciones de la demanda esta varia principalmente debido al sector en donde se encuentre teniendo en cuenta el producto o el servicio.

Las industrias relacionadas y de apoyo se refieren a la presencia o ausencia de industrias proveedoras y suplementarias que también sean competitivas internacionalmente

Finalmente la estrategia, estructura y rivalidad de las firmas determinan las condiciones que se requieren para la creación de una empresa que se quiera incluir dentro del sector.

Además del diamante de la competitividad en cuenta las cinco fuerzas competitivas mencionadas por (Porter ,2007,pag.69-95) como : la amenaza de entrada de nuevas empresas, la presión de productos sustitutos, poder negociador de los compradores,poder negociador de los proveedores, rivalidad entre los competidores existentes.

Desde la perspectiva industrial, el sector aeronáutico está considerado como «una industria de industrias» debido a la complejidad de como se integran los diferentes sectores para sacar un solo producto final, ya que muchas de estas empresa se encuentran ubicadas a miles de kilómetros la una y de la otra; sin embargo, al final tienen que unir la parte que cada una realiza para ensamblar el producto. En el caso específico de Colombia, se observa que, a pesar de tener uno de los mayores flujos aéreos en Latinoamérica, el desarrollo de su industria aeronáutica es bastante limitada y no corresponde con el gran mercado aeronáutico que posee el país.

En segundo lugar, para efectos de la investigación se adoptó un estudio cualitativo (Hernández, 2010), ya que el análisis se realizó de lo general a lo particular, lo que hace que no sea un estudio con un orden definido y que no se pueda generar hipótesis y preguntas a lo largo de toda la investigación. De hecho, el proceso de recolección de datos se puede dar de muchas formas dependiendo de los objetivos del autor.

Una de las ventajas del estudio cualitativo es que la recolección de datos y el proceso de análisis -del cual pueden surgir más preguntas o también conclusiones- se hacen de manera simultánea, y a partir de esta dinámica se va construyendo el diagnóstico. Además, con el estudio cualitativo se logra conocer los diferentes fenómenos y variables que rodean al clúster que, de manera directa o indirecta, influyen en su desarrollo. Este tipo de estudio también le da cabida a opiniones y perspectivas que pueden llevar a reflexionar en qué se ha acertado y en qué se ha fallado.

\section{Metodología}

La metodología propuesta es de tipo cualitativo, basatgfuda en la lógica del proceso inductivo, a saber: explorar, describir y generar perspectivas teóricas (Hernández, 2010). Por tanto, en este caso se utilizaron entrevistas abiertas a personas expertas, por su conocimiento y experiencia, que representan a los diferentes sectores del Clúster Aeroespacial en el Valle del Cauca. El método que se utilizó para hacer el estudio fue de análisis y síntesis, debido a que inicialmente se determinaron las fortalezas y debilidades que posee el Clúster Aeroespacial en el Valle del Cauca, y se identificaron los factores que han hecho que no haya tenido el éxito esperado.

Posteriormente, se efectuó una síntesis de la información recolectada para determinar cuáles han sido los aciertos y los desaciertos que se han cometido anteriormente y cómo se pueden revertir las fallas, teniendo como objetivo que las debilidades se conviertan en fortalezas y conservando o potencializando las fortalezas. La síntesis de la información se realizó a través del software Atlas TI, herramienta que fue fundamental para organizar la información y hacer las respectivas conclusiones.

Los resultados de la caracterización y análisis permitirán agregar valor al modelo de gestión que se espera que sea adoptado por el Clúster Aeroespacial en el Valle del Cauca para lograr su desarrollo.

\section{Desarrollo}

\section{Entrevistas a personas involucradas con el desarrollo del Clúster Aeroespacial del Valle del Cauca}

Se realizaron seis entrevistas a personas que se encuentran involucradas con el desarrollo del Clúster Aeroespacial en el Valle del Cauca y que representan los diferentes actores incluidos dentro de esta investigación.

\section{Conversatorio}

En el IV Encuentro Internacional de Administración en la Industria Aeronáutica Polo de Desarrollo Nacional se realizó un conversatorio entre seis expertos que están involucrados con el desarrollo del proyecto o que son conocedores del desarrollo de iniciativas clúster tanto a nivel nacional como internacional. 


\section{Matriz DOFA}

Después de haber recolectado toda la información a través de las entrevistas y del conversatorio, se realizó una matriz DOFA que ofrece una visión completa acerca de la situación actual en la que se encuentra el Clúster Aeroespacial en el Valle del Cauca.

\section{Debilidades}

Se debe que tener en cuenta que la industria aeroespacial es una de las más tecnificadas del mundo, esto hace que sea un sector extremadamente costoso; por tanto, para desarrollar un proyecto de este tipo «se requiere de un capital inmensamente grande, el cual solo posee el gobierno o una empresa multinacional que sea capaz de jalonar económicamente todo el proyecto» (Pérez, 2015). Teniendo en cuenta lo anterior las debilidades que actualmente posee el Clúster Aeroespacial en el Valle del Cauca son:

- D1- No se tiene el capital necesario para impulsar el proyecto de la manera que se requiere.

- D2- Existe una carencia de liderazgo que sea capaz de dirigir a todos los actores del clúster.

- D3- Hay una carencia de recursos humanos calificados que puedan llevar a cabo los proyectos.

- D4- Existe una falta de compromiso del Gobierno con el proyecto.

- D5- Se considera que hay un clúster cuando apenas se está trabajando en la agremiación o asociación.

- D6- No se posee una metodología ni unos objetivos claros que permitan que todos trabajen de una manera eficiente y coordinada.

- D8- El proyecto no se ha articulado con otras iniciativas clúster que puedan apoyar y ser socios del proyecto.

- D9- Es latente la falta de confianza entre los diferentes actores del clúster.

- D10-Gran cantidad de los productos del clúster no se encuentran certificados.

\section{Fortalezas}

La privilegiada posición geográfica del Valle del Cauca y el conglomerado de industria metalmecánica que hay en la región hace que esta se vuelva ideal para desarrollar un clúster aeronáutico (Arroyo, 2015). Además, la región ha empezado un proceso de desarrollo dirigido hacia la formación de un mercado mucho más dinámico, con una industria mucho más eficiente, que ha hecho que inversionistas y compañías extranjeras empiecen a ver, cada vez más, al Valle del Cauca como una región más atractiva. Lo anterior le brinda una serie de fortalezas las cuales son:

- F1- La privilegiada posición geográfica del Valle del Cauca hace que sea una zona muy atractiva para el emprendimiento económico.

- F2- Las principales compañías involucradas dentro del clúster tienen una gran experiencia dentro del sector aeronáutico.

- F3- Las principales productoras de aeronaves en Colombia se han dejado de ver como competencia y se han comenzado a agremiar.

- F4- El desarrollo de las comunicaciones hace que sea muy fácil la comunicación entre los diferentes actores del proyecto.

- F5- La industria colombiana ha sido capaz de copiar muchos modelos productivos de manera exitosa.

\section{Oportunidades}

Hay condiciones de favorabilidad, pero es pertinente saberlas aprovechar; para esto hay que generar unas condiciones óptimas que faciliten que estas oportunidades empiecen a producirle beneficios al proyecto, de una manera rápida y efectiva. Actualmente, las principales oportunidades que se presentan al Clúster Aeroespacial en el Valle del Cauca son:

- O1- Incluirse dentro del paquete de proyectos de emprendimiento económico que apoya el Gobierno.

- O2- Desarrollar programas en universidades que suplan los recursos humanos requeridos por el clúster -existe un importante número de universidades en donde podría hacerse - .

- O3- Impulsar la investigación a través de la vinculación de la academia y la experiencia que puede brindar la Fuerza Aérea Colombiana.

- O4-Tomar en cuenta otras iniciativas clúster de un sector similar al aeronáutico que podrían apoyar el desarrollo del proyecto y servir como socios.

\section{Amenazas}

Algunos factores como la influencia política, en este tipo de proyectos, pueden ser contraproducentes debido a que hay prioridades en las agendas de los gobernantes que en muchas ocasiones no incluye la designación de recursos a proyectos de gestión tecnológica ni de emprendimiento industrial (Torres, 2015). Por otro lado, están las diferentes dinámicas de la economía y las variables socioculturales que pueden influir de manera negativa en el 
desarrollo del proyecto. Las principales amenazas que actualmente acechan al Clúster Aeroespacial en el Valle del Cauca son:

- A1-La influencia política dentro del proyecto puede dar cabida a retrasos y falta de continuidad debido a que en cada administración las prioridades de la agenda cambian.

- A2- Aún hay muchos sectores económicos y gubernamentales de la región que creen que el proyecto todavía no tiene cabida.

- A3- Las barreras jurídicas pueden retrasar aún más el desarrollo del proyecto.

- A4- La situación económica actual del país no es la mejor, como consecuencia los recursos para invertir son bastante limitados.

- A5- En la Cámara de Comercio hay un exceso de propuestas de emprendimiento industrial (Arroyo, 2015).

\section{Estrategias}

A continuación se presentan una serie de estrategias a través de las cuales se busca potenciar las fortalezas, plantear maneras de crear nuevas oportunidades, crear métodos para eliminar las debilidades y, por último, visualizar amenazas y crear mecanismos para protegerse de ellas.

- Estrategias DA. En el ámbito económico, las debilidades del clúster se basan en la falta de capacidad económica de los actores que hacen parte tanto de la pequeña como de la mediana industria (Arrazola, 2015); esto implica que para llevar a cabo un proyecto tan costoso como lo sería el Clúster Aeroespacial en el Valle del Cauca, de acuerdo a la estructura que se tiene pensada, solo será posible con:

D1A2- Conseguir la ayuda del Estado o la inversión de grandes multinacionales que por sí sola pueda jalonar el proyecto.

D2A2- Contrarrestar los sentimientos de desconfianza que se presentan entre los diferentes actores del clúster, estableciendo mecanismos que integren y comprometan a las diferentes partes y fomentando un ambiente de confianza.

D4A2- Ofrecerle facilidades y beneficios a compañías, tanto nacionales como extranjeras, que puedan incluirse $y$ aportarle al proyecto.
D10A5- Realizar un gran trabajo de gestión tecnológica que le permita al proyecto trabajar con tecnología de punta que contribuya a la certificación de sus productos.

- Estrategia FA. La falta de compromiso, el desconocimiento del proyecto, la carencia de recursos humanos y la falta de capital son variables que se deben suplir a través de la experiencia, del aprovechamiento de un crecimiento del sector aeronáutico y de una privilegiada posición geográfica. Esto se puede subsanar al:

F1A2- Conseguir una entidad que facilite capital a través del Gobierno o de entidades multinacionales, que puedan suplir esa falta de recursos que actualmente tiene el proyecto, debido a que los actores pertenecen a la pequeña y mediana industria.

F3A2- Crear estrategias que promocionen el clúster desde el ámbito regional hasta el internacional, para evitar ese desconocimiento y desinterés que tiene el proyecto en sectores tan importantes como el gubernamental, industrial y académico.

F5A3- Tener en cuenta que, a pesar de que sus productos no están certificados, muchas de las compañías que desarrollan y fabrican estos productos tienen más de cuarenta años de experiencia.

- Estrategia Do. Teniendo en cuenta los pocos recursos, puede aprovecharse el apoyo del Gobierno y articularse con otras iniciativas clúster como la automotriz (Arroyo, 2015) para poder ser más competitivos, por lo tanto se debe tener en cuenta las siguientes estrategias:

D804- A pesar de que la industria aeronáutica en el país ha tenido poco desarrollo, se puede aprovechar el hecho de que a nivel mundial este sector está teniendo un gran crecimiento debido a que es el medio de transporte más efectivo; esto hace que el proyecto tenga un gran potencial a mediano y largo plazo.

D4O1 - Actualmente, el Gobierno ha empezado a ver el potencial que tiene el clúster como modelo de desarrollo industrial, esto hace que, sin que todavía exista un marco legal muy claro para este tipo de asociaciones, sí se pueda sacar provecho de instituciones estatales como Red Clúster Colombia que asesoran y buscan nuevas oportunidades para este tipo de iniciativas clúster (Caicedo, 2015). 
CIENCIA Y PODER AÉREO | ISSN 1909-7050 | E-ISSN 2389-9468 | Vol. 12 | Ene - Dic 2017 | Escuela de Postgrados de la Fuerza Aérea Colombiana | pp 60-70

D1003- Debido al poco desarrollo tecnológico aeronáutico en el país, instituciones como las Fuerzas Militares -dentro de sus varios contratos de compra, mantenimiento y modernización de sus aeronaves- han firmado convenios de transferencia de tecnología con importantes empresas como lo es la Embraer, lo que hay que aprovechar porque sería muy útil para el desarrollo de las compañías y del clúster en general.

- Estrategia FO. Al tener una posición tan privilegiada geográficamente, en un sector que crece y se diversifica cada vez más, el clúster podría atraer muchos inversionistas, tanto nacionales como extranjeros. Al respecto, las estrategias serían:

F304- Agremiar a las compañías pertenecientes al clúster, debido a que se hace más fácil trabajar de manera conjunta y se puedan acceder a las ayudas del gobierno.

F404- Se crearán mecanismos de comunicación más efectivos, ya que con las grandes facilidades de comunicación que posee el mundo actual se hace mucho más fácil una efectiva comunicación entre todos los actores del clúster.

F501-La industria colombiana, a lo largo de la historia, ha copiado de manera exitosa muchos elementos y modelos del exterior, al lograr adaptarlos a las necesidades y al entorno nacional (González,2015).

En la Tabla 1 se muestra de forma organizada la matriz DOFA con el resultado de las estrategias.

Tabla 1.

Matriz DOFA

\begin{tabular}{|c|c|c|}
\hline MATRIZ DOFA & FORTALEZAS & DEBILIDADES \\
\hline & $\begin{array}{l}\text { La privilegiada posición geográfica del } \\
\text { Valle del Cauca hace que sea una zona muy } \\
\text { atractiva para el emprendimiento económico. }\end{array}$ & $\begin{array}{l}\text { No se tiene el capital necesario para impulsar el } \\
\text { proyecto de la manera que se requiere. }\end{array}$ \\
\hline & $\begin{array}{l}\text { Las principales compañías involucradas con } \\
\text { el clúster tienen una gran experiencia dentro } \\
\text { del sector aeronáutico. }\end{array}$ & $\begin{array}{l}\text { Existe una carencia de liderazgo que sea capaz } \\
\text { de dirigir todos los actores del clúster. }\end{array}$ \\
\hline & $\begin{array}{l}\text { Las principales productoras de aeronaves } \\
\text { en Colombia se han dejado de ver como } \\
\text { competencia y se han comenzado a agremiar. }\end{array}$ & $\begin{array}{l}\text { Carencia de recursos humanos calificados que } \\
\text { puedan llevar a cabo los proyectos adelante. }\end{array}$ \\
\hline & $\begin{array}{l}\text { El desarrollo de las comunicaciones hace } \\
\text { que sea muy fácil la comunicación entre los } \\
\text { diferentes actores del proyecto. }\end{array}$ & $\begin{array}{l}\text { La falta de compromiso del Gobierno con el } \\
\text { proyecto. }\end{array}$ \\
\hline & $\begin{array}{l}\text { La industria colombiana ha sido capaz de } \\
\text { copiar muchos modelos productivos de } \\
\text { manera exitosa. }\end{array}$ & $\begin{array}{l}\text { Se considera que existe un clúster cuando } \\
\text { apenas se está trabajando en su agremiación. }\end{array}$ \\
\hline OPORTUNIDADES & ESTRATEGIAS FO & ESTRATEGIAS DO \\
\hline $\begin{array}{l}\text { Incluirse dentro del paquete de } \\
\text { proyectos de emprendimiento } \\
\text { económico que apoya el } \\
\text { Gobierno. }\end{array}$ & $\begin{array}{l}\text { Al tener una posición tan privilegiada } \\
\text { geográficamente, el clúster podría atraer } \\
\text { muchos inversionistas tanto nacionales como } \\
\text { extranjeros. }\end{array}$ & $\begin{array}{l}\text { Teniendo en cuenta los pocos recursos, se } \\
\text { puede aprovechar el apoyo del gobierno y la } \\
\text { articulación con otras iniciativas Clúster como la } \\
\text { automotriz para poder volverse más competitivo. }\end{array}$ \\
\hline
\end{tabular}

66 | ICaracterización del clúster aeroespacial en el Valle del Cauca aplicando el método cualitativo que permita identificar las estrategias de gestión competivas 


\begin{tabular}{|c|c|c|}
\hline $\begin{array}{l}\text { Existe un importante número } \\
\text { de universidades en donde se } \\
\text { podrían desarrollar programas } \\
\text { que suplan los recursos humanos } \\
\text { requeridos por el Clúster. }\end{array}$ & $\begin{array}{l}\text { Cuando existe una agremiación de las } \\
\text { compañías pertenecientes al clúster, se hace } \\
\text { más fácil trabajar de manera conjunta y se } \\
\text { puede acceder a las ayudas del Gobierno. }\end{array}$ & $\begin{array}{l}\text { A pesar de que la industria aeronáutica en el } \\
\text { país casi no está desarrollada, mundialmente } \\
\text { este sector está teniendo un gran crecimiento } \\
\text { debido a que es el medio de transporte más } \\
\text { efectivo, esto hace que el proyecto tenga un } \\
\text { gran potencial a mediano y largo plazo. }\end{array}$ \\
\hline $\begin{array}{l}\text { Impulsar la investigación a } \\
\text { través de la vinculación de la } \\
\text { academia y la experiencia que } \\
\text { puede brindar la Fuerza Aérea } \\
\text { Colombiana. }\end{array}$ & $\begin{array}{l}\text { Con las grandes facilidades de comunicación } \\
\text { que posee el mundo actual se hace } \\
\text { mucho más fácil que exista una efectiva } \\
\text { comunicación entre todos los actores del } \\
\text { clúster. }\end{array}$ & $\begin{array}{l}\text { Actualmente el Gobierno ha empezado a ver el } \\
\text { potencial que tiene el clúster como modelo de } \\
\text { desarrollo industrial, lo que hace que, incluso sin } \\
\text { que todavía haya un marco legal muy claro para } \\
\text { este tipo de asociaciones, sí existan instituciones } \\
\text { estatales como Red Clúster Colombia que } \\
\text { asesoran y buscan nuevas oportunidades para } \\
\text { este tipo de iniciativas clúster. }\end{array}$ \\
\hline $\begin{array}{l}\text { Existen otras iniciativas clúster de } \\
\text { un sector similar al aeronáutico } \\
\text { que podrían apoyar el desarrollo } \\
\text { del proyecto y servir como } \\
\text { socios. }\end{array}$ & $\begin{array}{l}\text { La industria colombiana, a lo largo de la } \\
\text { historia, ha copiado de manera exitosa } \\
\text { muchos elementos y modelos del exterior, } \\
\text { al lograr adaptarlos a las necesidades y al } \\
\text { entorno nacional. }\end{array}$ & $\begin{array}{l}\text { Debido al poco desarrollo tecnológico } \\
\text { aeronáutico en el país, instituciones como las } \\
\text { Fuerzas Militares - dentro de sus varios contratos } \\
\text { de compra, mantenimiento y modernización } \\
\text { de sus aeronaves- han firmado convenios de } \\
\text { transferencia de tecnología con importantes } \\
\text { empresas como lo es la Embraer, lo que sería } \\
\text { muy útil para el desarrollo de las compañías y del } \\
\text { clúster en general. }\end{array}$ \\
\hline AMENAZAS & ESTRATEGIA FA & ESTRATEGIAS DA \\
\hline $\begin{array}{l}\text { La influencia política dentro del } \\
\text { proyecto puede dar cabida a } \\
\text { retrasos y falta de continuidad } \\
\text { debido a que en cada } \\
\text { administración las prioridades de } \\
\text { la agenda cambian. }\end{array}$ & $\begin{array}{l}\text { A través del apoyo económico del Gobierno } \\
\text { o de una gran multinacional, se podrá suplir } \\
\text { esa falta de recursos que actualmente tiene el } \\
\text { proyecto debido a que los actores pertenecen } \\
\text { a la pequeña y mediana industria. }\end{array}$ & $\begin{array}{l}\text { El Gobierno debe crear unas condiciones legales } \\
\text { especiales que le brinden beneficios y facilidades } \\
\text { al clúster. Por otra parte, los actores del clúster } \\
\text { deben mostrar el compromiso por cumplir todos } \\
\text { los requerimientos que exige el gobierno. }\end{array}$ \\
\hline $\begin{array}{l}\text { Aún existen muchos sectores } \\
\text { económicos y gubernamentales } \\
\text { de la región que creen que el } \\
\text { proyecto todavía no tiene cabida. }\end{array}$ & $\begin{array}{l}\text { Hay que crear estrategias que promocionen } \\
\text { el clúster desde el ámbito regional hasta el } \\
\text { internacional, para evitaresedesconocimiento } \\
\text { y desinterés que tiene el proyecto en sectores } \\
\text { tan importantes como el gubernamental, el } \\
\text { industrial y el académico. }\end{array}$ & $\begin{array}{l}\text { Para contrarrestar esos sentimientos de } \\
\text { desconfianza que hay entre los diferentes actores } \\
\text { del clúster, se deben establecer mecanismos } \\
\text { que integren y comprometan a las diferentes } \\
\text { partes, para así crear un ambiente de confianza } \\
\text { en donde todos tengan la certeza de que cada } \\
\text { uno está haciendo todo lo posible a través de } \\
\text { sus capacidades para poder sacar adelante este } \\
\text { proyecto. }\end{array}$ \\
\hline $\begin{array}{l}\text { Las barreras jurídicas pueden } \\
\text { retrasar aún más el desarrollo } \\
\text { del proyecto. }\end{array}$ & $\begin{array}{l}\text { Actualmente el gobierno está apoyando } \\
\text { las iniciativas Clúster, la clave se encuentra } \\
\text { en que el Clúster Aeroespacial en el Valle } \\
\text { del Cauca consiga incluirse dentro de las } \\
\text { iniciativas que están siendo apoyadas por el } \\
\text { gobierno a través de asesorías y recursos. }\end{array}$ & $\begin{array}{l}\text { Para ser un clúster exitoso, se necesita un gran } \\
\text { trabajo de gestión tecnológica que le permita al } \\
\text { proyecto trabajar con tecnología de punta, que } \\
\text { contribuya a la certificación de sus productos } \\
\text { por parte de las autoridades aeronáuticas, } \\
\text { tanto nacionales como internacionales. Esto es } \\
\text { fundamental para poder estar dentro de uno de } \\
\text { los sectores más tecnificados del mercado. }\end{array}$ \\
\hline
\end{tabular}

Fuente. elaboración propia.

\section{Propuesta de Gestión}

En esta propuesta de gestión para el Clúster Aeroespacial en el Valle del Cauca se mencionan las acciones, y la forma en la que deben realizarse, para darle un impulso al proyecto, que genere las condiciones necesarias para que este empiece a sobresalir y, por consiguiente, para que se promueva su desarrollo para beneficiar al Valle del Cauca, a la Fuerza Aérea Colombiana y al sector aeronáutico nacional. 


\section{Segmentos de clientes}

Se determinaron las principales líneas de clientes del Clúster Aeroespacial en el Valle del Cauca. El primero son las Fuerzas Armadas de Colombia que, debido a la situación de orden público de las últimas décadas, poseen una gran flota que requiere mantenimiento y actualización constante.

Por otro lado, se encuentran las aerolíneas comerciales, tanto nacionales como internacionales, que ven a Colombia como la entrada a Suramérica, lo que crea la necesidad de tener un flujo cada vez mayor de aeronaves y, por consiguiente, todos los productos y servicios que requiere el tener una flota en un alto nivel de disponibilidad. Aquí cabe mencionar a los aviones corporativos y recreativos, así como los aviones de instrucción y fumigación.

\section{Propuestas de venta}

Cada producto que desarrolle el clúster debe tener una adecuada publicidad dentro de los potenciales clientes, además, debe crear la necesidad del producto en la mayor cantidad de compañías y empresarios. Es importante anotar que debe pasar un tiempo mientras el clúster se posiciona en el mercado y empiece a retribuir toda la inversión que se reciba.

\section{Canales de distribución y comunicación}

Lo primero que debe hacerse es estandarizar los canales de distribución y comunicación para que todos los miembros de la organización tengan claro cuáles son y de qué forma los puedan utilizar. Así se evitarán confusiones y demoras innecesarias. En segunda medida, deben crearse mecanismos de verificación y cualificación constante que permitan estar mejorando cada día estos canales.

Además de lo anterior, se deben propiciar las condiciones necesarias para que estos mecanismos funcionen, entre ellas, una cercanía geográfica entre las compañías, unos buenos sistemas de comunicación entre las compañías, la infraestructura y los medios de transporte necesarios para permitir una industria eficiente y efectiva, $y$, por último y más importante, tener una disposición de colaboración y trabajo en equipo.

\section{Relación con el cliente}

Para el éxito del clúster es necesaria una excelente relación con el cliente, tanto antes como después de la compra. Antes de la adquisición, para poder mostrarle al cliente la calidad y los beneficios que pueden ofrecer tanto los productos como los servicios, procurando convencerlo de hacer su compra e incluso de aumentar la cantidad de esta. Después de la adquisición, para que el cliente adquiera todos los servicios de mantenimiento y actualización con las compañías del clúster y cuando requiera un nuevo producto lo realicen con ellas.

\section{Flujos de ingreso y egreso}

Mantener unos estados financieros organizados y controlados es algo fundamental para tener un control estricto de los recursos, tanto de los que entran como de los que salen de la organización. Esto permite hacer una planificación de los proyectos que se pueden desarrollar y de los recursos con los que se cuenta para cada uno de ellos. Por otra parte, se podrá evidenciar el nivel de crecimiento o decrecimiento del clúster y, además, se podrá determinar cuáles son los recursos que pagarán los costos y deudas, cuáles son los recursos que se van a reinvertir y los que serán repartidos entre los accionistas.

\section{Recursos clave}

Dentro del clúster debe haber una jerarquización de los recursos tanto monetarios como físicos y obviamente esta jerarquización debe estar estandarizada entre todos los actores del clúster, de esta forma no serán mal invertidos los recursos clave.

\section{Actividades clave}

A pesar de que la experiencia no ha sido exitosa, es vital que se rescate y se aprenda acerca de las fallas que se presentaron en el proceso, así como de los aciertos, para a partir de allí determinar cuál es el mejor proceso a desarrollar. Para lo anterior, es importante tener en cuenta las siguientes actividades e información claves:

- Lograr convencer al Gobierno, universidades, empresas y al público en general de que el Clúster Aeroespacial en el Valle de Cauca es un proyecto con gran potencial que le puede traer grandes beneficios a la región.

- Definir lo más pronto posible las empresas y universidades que se involucrarán dentro del proyecto.

- Es necesario formar una asociación efectiva entre todos los actores de la empresa que lo que busque sea sumar fortalezas y minimizar debilidades.

- Estandarizar diferentes métodos y procedimientos de comunicación para facilitar el trabajo entre los diferentes actores del clúster.

- Cuando se empiecen a desarrollar los ciclos productivos dentro del clúster, se debería tener una importante base tecnológica que asegure la realización de cadenas productivas que generen productos de buena calidad a bajos costos.

- La empresas que pertenezcan al clúster deberán crear una comisión de recursos humanos con una 
estrategia de comunicación que permita informar a las universidades sobre cuáles son los requerimientos de educación que demandan las empresas.

- La universidad deberá empezar a contribuir con el desarrollo tecnológico del clúster a través de sus centros de investigación.

- Para incentivar la participación de las universidades se requiere participar en las convocatorias de ciencia, tecnología e innovación de las diferentes entidades públicas y privadas.

- Recolectar los documentos y exigencias requeridas por el gobierno para que el proyecto quede plenamente legalizado y apto para recibir los beneficios que ofrece el Gobierno a través de las convocatorias innovación e iniciativas clúster.

- Después de conformado y legalizado el clúster, realizar un plan estratégico y un estudio de mercado donde se determine cuáles son los posibles proyectos a desarrollar dependiendo de la demanda del mercado.

- Para planificar toda la estrategia y organización que requiere el clúster es necesario tener uno o varios expertos en el ámbito de Gestión Tecnológica e Innovación, que asesoren, guíen y acompañen el proceso de desarrollo del clúster.

- Dentro del plan de acción, tener en cuenta la metodología que se va a utilizar como la de rutas competitivas, dado que ya ha tenido éxito en clústeres internacionales e incluso nacionales, y además es el que recomienda el Gobierno para el desarrollo de este tipo de iniciativas.

- Aprovechar el poder de articulación de la Fuerza Aérea Colombiana, una institución de mucho prestigio que, además de ser un cliente potencial del clúster, es capaz de reunir a los diferentes actores del clúster y ayudar tanto con sus procesos técnicos como con los organizativos.

- Para facilitar el trabajo en equipo del clúster, ubicar a los actores del clúster con una cercanía geográfica para facilitar la comunicación y reducir costos de producción en cuanto al transporte de materias primas y ensamble, así como a la distribución del producto.

- Es de vital importancia que se maximicen los recursos e instalaciones que nos brinda el Gobierno, ya que estos son bastante limitados y no se dan de manera continua o indefinida.
- El Gobierno también es un elemento vital para promocionar este tipo de proyectos tanto a nivel nacional como internacional, además de servir como ente mediador y facilitador en todo tipo de acuerdos que se puedan realizar.

- Después de conformado el clúster, es necesario realizar alianzas estratégicas con otros clústeres de la región, como lo es el de transporte y el energético, que ayudarían a aumentar las capacidades para la construcción de aeronaves o partes de estas cada vez más complejas y de una manera más efectiva y eficiente.

- La industria aeronáutica, al ser tan grande y tener tantos subsectores, se caracteriza por tener grandes multinacionales que tienen presencia en todos esos subsectores y que abarcan gran parte del mercado.

- Para certificar tanto las aeronaves como las partes que actualmente producen las compañías del clúster, y las que producirá en un futuro, es necesario asistir a todos los talleres de capacitación que ofrece el Gobierno para certificarlos frente a autoridades nacionales e internacionales.

\section{Socios Estratégicos}

En Latinoamérica hay importantes compañías y clúster del sector aeronáutico con los que se pueden firmar diferentes acuerdos de colaboración, trabajo en conjunto y transferencia de tecnología para ir aumentando las capacidades y posibilidades de negocio del Clúster Aeroespacial en el Valle del Cauca.

Dentro de las más destacadas, se encuentran compañías como Embraer que tiene una importante presencia de aeronaves a nivel nacional e internacional y con la que, a través del gobierno o compañías comerciales, se pueden firmar contratos de cooperación industrial asociada, donde se contemple la transferencia de tecnología a la industria colombiana, cuando compren sus productos o adquieran los servicios de esta compañía.

Esto ayudaría sustancialmente a reducir la brecha tecnológica entre la industria colombiana y las potencias mundiales, además de proporcionar la información necesaria para desarrollar un clúster igual de eficiente y efectivo al de las organizaciones que están transfiriendo sus conocimientos.

\section{Conclusiones}

Con esta investigación se pretende que los actores involucrados apliquen las recomendaciones y estrategias 
CIENCIA Y PODER AÉREO | ISSN 1909-7050 | E-ISSN 2389-9468 | Vol. 12 | Ene - Dic 2017 | Escuela de Postgrados de la Fuerza Aérea Colombiana | pp 60-70

que conduzcan a posicionar el clúster a nivel nacional e internacional, y que contribuya al desarrollo, social, económico y académico del Valle del Cauca.

Como resultado de la investigación se aportan en términos generales ciertas recomendaciones y observaciones. En primer lugar, el apoyo del Gobierno es un elemento indispensable para el desarrollo del proyecto, debido a que es un elemento que sirve de articulador entre los diferentes actores del clúster, además de ser el único capaz hasta el momento de darle el impulso económico que requiere. Aparte del apoyo financiero, el Gobierno puede brindar muchas facilidades tanto legales como jurídicas, y asesorías tanto técnicas como organizativas, que facilitarían la reducción, en gran medida, de los costos del proyecto y también contribuirían al posicionamiento de una imagen corporativa más atractiva del proyecto frente a nuevos inversores, tanto nacionales como extranjeros.

En segundo lugar, para la Fuerza Aérea Colombiana el desarrollo del Clúster Aeroespacial del Valle del Cauca, al igual que los otros clústeres que se están desarrollando a nivel nacional, es una inmensa oportunidad para reducir costos de mantenimiento y operación de sus aeronaves, además de la posibilidad de desarrollar en conjunto con la industria nacional nuevas aeronaves y diferentes tipos de actualizaciones para las que ya posee. Lo anterior aumentaría sustancialmente sus capacidades operativas para poder cumplir con su misión.

En tercer lugar, la academia, actualmente, no tiene los programas necesarios para poder suplir la demanda de recursos humanos que se requieran en caso de un posible desarrollo del clúster, y los centros de investigación de las universidades todavía no se han vinculado de manera efectiva con el proyecto, desconociendo una importante fuente de desarrollo tecnológico.

Por otra parte, la industria aeronáutica es uno de los sectores más atractivos que actualmente existe en el mercado debido a que es una industria que genera miles de millones de dólares en utilidades y que ha tenido mayores niveles de crecimiento en las dos últimas décadas.

Por último, si se pretende que Colombia se introduzca de una manera significativa en la industria aeronáutica, es necesario que no solo se trabaje en el Clúster Aeroespacial en el Valle del Cauca, sino que posteriormente se trabaje en un gran clúster nacional que reúna a todos los clústeres aeronáuticos del país, con el objetivo de unir las fortalezas de cada uno y poder, así, aumentar las capacidades del país para desarrollar productos y servicios de mayor complejidad que puedan posicionarse a nivel mundial.

\section{REFERENCIAS}

\section{Acosta, J. (2012). ¿Es la politica de competitividad e innovación la nueva política industrial de colombia? Bogotá: Fescol.}

Arrazola, I. (2015). Caracterización del Clúster Aeroespacial en el Valle del Cauca aplicando el método cualitativo (D. Morante Granobles, entrevistador).

Arroyo, S. (2015). Caracterización del Clúster Aeroespacial en el Valle del Cauca aplicando el método cualitativo (D. Morante Granobles, entrevistador).

Caicedo, A. (2015). IV Encuentro Internacional de Administración en la Industria Aeronaútica Polo de Desarrollo Nacional (D. Morante Granobles, Entrevistador).

Gonzalez, M. (2015). Caracterización del Clúster Aeroespacial en el Valle del Cauca aplicando el método cualitativo (D. Morante Granobles, entrevistador).

Hernández, R., Fernández, C. \& Baptista. (2010). Metodología de la investigación. México D.F: McGraw-Hill/ Interamericana Editores S.A. DE C.V.

Perego, L. (2003). Competitividad a partir de las agrupaciones industriales. Argentina: Facultad de Ciencias Económicas de la Universidad de la Plata.

Perez, F. (2015). IV Encuentro Internacional de Administración en la Industria Aeronáutica Polo de Desarrollo Nacional (D. Morante Granobles, entrevistador).

Porter, M. (2007). La ventaja competitiva de las naciones. Massachusetts: Harvard Bussiness School Publishing.

Torres, D. (2015). Caracterización del Clúster Aeroespacial en el Valle del Cauca aplicando el método cualitativo (D. Morante Granobles, entrevistador). 


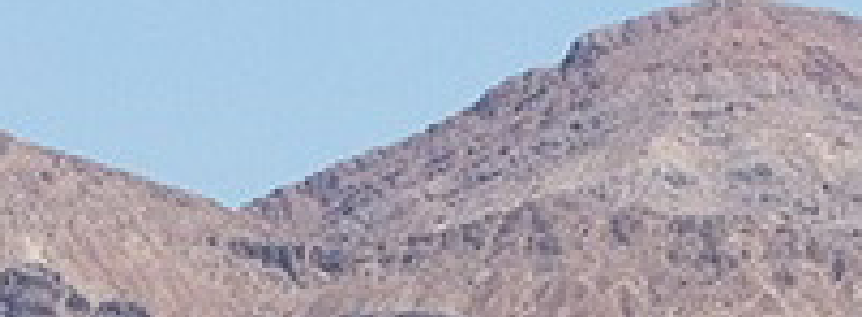

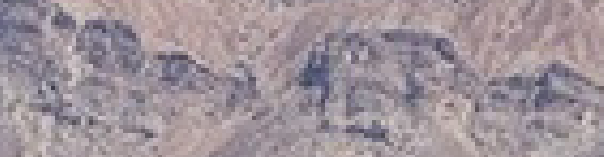

